\title{
Wave velocities in a pre-stressed anisotropic elastic medium
}

\author{
M D Sharma ${ }^{1, *}$ and NeEtu Garg ${ }^{2}$ \\ ${ }^{1}$ Department of Mathematics, Kurukshetra University, Kurukshetra 136 119, India. \\ ${ }^{2}$ UIET, Kurukshetra University, Kurukshetra 136 119, India. \\ *e-mail: mohan_here@rediffmail.com
}

\begin{abstract}
Modified Christoffel equations are derived for three-dimensional wave propagation in a general anisotropic medium under initial stress. The three roots of a cubic equation define the phase velocities of three quasi-waves in the medium. Analytical expressions are used to calculate the directional derivatives of phase velocities. These derivatives are, further, used to calculate the group velocities and ray directions of the three quasi-waves in a pre-stressed anisotropic medium. Effect of initial stress on wave propagation is observed through the deviations in phase velocity, group velocity and ray direction for each of the quasi-waves. The variations of these deviations with the phase direction are plotted for a numerical model of general anisotropic medium with triclinic/ monoclinic/orthorhombic symmetry.
\end{abstract}

\section{Introduction}

In civil engineering and geophysics, the problems of consolidation and tectonics involve earth masses that are initially under high stress. The folds and fractures in the sedimentary layers are the result of differential stress environment in the sediments. In the problems of foundation engineering, the influence of initial stress appears in a buoyancy effect which amounts to floating a building on its foundation. The theory of elastic wave propagation in pre-stressed solids has a nearly two-century long history. In perhaps, the earliest effort Cauchy (1827) assumed that the initial stress was due to the central forces between the particles of solid. A definitive theory explaining the elastodynamics of a body under initial stress was developed by Biot $(1939,1940)$. An elegant and elaborate exposition of this theory is found in Biot (1965). Hayes (1963) studied the velocities of wave propagation in pre-stressed elastic solids. A large number of studies in the later years improved the understanding of wave propagation characteristics of materials under initial stress. Tolstoy (1982) studied the effect of gravity and hydrostatic pressure on velocities of elastic waves. Sharma and Gogna (1991) studied the effect of initial stress on Love waves in a sandy layer over a poroelastic solid. Degtyar and Rokhlin (1995, 1997) discussed the stress effects on ultrasonic wave velocities. In another study, Degtyar and Rokhlin (1998) discussed the effect of initial stress on wave propagation through an anisotropic/anisotropic interface. They used the stress dependent orthotropic elastic coefficients from a study by Man and Lu (1987).

Elastic anisotropy is a widespread observation in the areas of economic and scientific interest (Crampin 1987). Crampin (1994) reviewed the various studies related to the observation of shearwave splitting and confirmed that anisotropy was present in almost all the rocks in the uppermost half of the crust. On the other hand, the slow process of creep inside the earth produces the initial stress which may be approximated as homogeneous near the surface (Biot 1940). This implies the co-existence of elastic anisotropy as well as initial stress in the crust. An isotropic medium under initial stress behaves anisotropic to wave propagation.

Keywords. Pre-stress; anisotropy; phase velocity; group velocity.

J. Earth Syst. Sci. 115, No. 2, April 2006, pp. 257-265

(C) Printed in India. 
This may imply that the presence of initial stress in the earth masses is a cause for seismic anisotropy. Otherwise, the preferential alignments in the Earth ranging from mineral orientations, grains, or microcracks to regional fractures result in the seismic anisotropy. The effect of initial stress on waves cannot be accounted by the elastic anisotropy or the change in elastic constants and, hence, the existence of initial stress opens a new aspect in the nature of wave propagation (Biot 1940). It provides motivation to study the wave propagation in an elastic material with its elastic anisotropy supplemented with the presence of initial stress. Norris and Sinha (1995) studied the effects of anisotropy and pre-stress on the wave propagation along a fluid-solid interface. Surface wave velocities in a pre-stressed anisotropic solid is determined by Chai and Wu (1996). In a recent study, Prikazchikov and Rogerson (2003) analysed the dynamic properties of anisotropic pre-stressed elastic solids.

The presence of initial stresses affects the velocities, amplitudes and propagation directions of waves in a medium. In anisotropic media energy travels with group velocity along a ray at an angle to the propagation direction. It is the group velocity that is measured in observations of arrival times. The group velocity is also required for the interpretation of both real and synthetic data. The group velocity and ray direction of a wave is derived from its phase velocity that appears in equation of motion and most other analytical expressions. The work presented studies the effect of initial stress on the phase velocity, group velocity and ray direction in a general anisotropic elastic medium.

\section{Anisotropic elastic medium under initial stress}

\subsection{Equation of motion}

Consider a general anisotropic elastic medium under homogeneous initial stress $S_{i j}$. Following Biot (1965), the constitutive equations for wave motion in this medium, in the absence of body force, are given by

$$
s_{i j, j}+S_{j k} \omega_{i k, j}+S_{i k} \omega_{j k, j}=\rho \ddot{u}_{i},
$$

where $u_{i}$ are displacement components. The components of rotation are given by $\omega_{i j}=\left(u_{i, j}-u_{j, i}\right) / 2$. Indices can take values of $1,2,3$. The comma before an index represents (partial) space differentiation and dot denotes (partial) time derivative. Repeated index implies summation. The incremental stresses $s_{i j}$ in the medium are expressed as

$$
s_{i j}=B_{i j k l} u_{k, l}
$$

where, the fourth rank tensor $B_{i j k l}\left(=B_{j i k l}=\right.$ $\left.B_{i j l k}\right)$ represents the elastic properties of the medium. $\rho$ is the density of the medium. Another property, given by

$$
B_{i j k l}-B_{k l i j}=S_{k l} \delta_{i j}-S_{i j} \delta_{k l},
$$

ensures the existence of energy density function for the medium (Biot 1939, 1965), where $\delta_{i j}$ is Kronecker delta.

\subsection{Plane wave propagation}

To seek the harmonic solution of (1), for the propagation of plane waves, write

$$
u_{j}=U_{j} \exp \left\{\imath\left(\frac{1}{v} n_{k} x_{k}-t\right)\right\}, \quad(j=1,2,3),
$$

where $\omega$ is angular frequency. $v$ is the phase velocity of the wave, along the phase direction $\left(n_{1}, n_{2}, n_{3}\right)$. Substituting (4) in (1) yields a system of three homogeneous equations, given by

$$
\left(X_{i k}-h \delta_{i k}\right) U_{k}=0, \quad(i=1,2,3),
$$

where, for row matrix $\mathbf{N}=\left(n_{1}, n_{2}, n_{3}\right)$ and its transpose $\mathbf{N}^{T}$,

$$
h=v^{2}-\frac{\mathbf{N S N}^{T}}{\rho},
$$

and $X_{i k}$ are the elements of a matrix

$$
\mathbf{X}=\frac{\left[\mathbf{Z}-\mathbf{S}+\mathbf{S N}^{T} \mathbf{N}-\mathbf{N}^{T} \mathbf{N S}\right]}{\rho} .
$$

The matrix $\mathbf{S}=\frac{1}{2}\left\{S_{i j}\right\}$ and matrix $\mathbf{Z}$ is as defined in Appendix A. However, the matrix $\mathbf{Z}$ is not a symmetric matrix but due to the relations (3), the matrix $\mathbf{X}$ is a symmetric one. The system (5) may be called the modified Christoffel equations. The phase velocity $v$ is related to the eigenvalue $h$ of this system through an expression, given by

$$
\left.v=\sqrt{(} h+\mathbf{N S N}^{T} / \rho\right) .
$$

It may be noted that on replacing the matrix $Z$ with $C_{i j k l} n_{j} n_{l}$, the Christoffel equations (5) will be the same as in Degtyar and Rokhlin (1998), obtained by using the theory developed by Man and Lu (1987). From the above relations, it is deduced that for the values of $\mathbf{N}$ and initial stress components giving $\mathbf{S N}^{T} \mathbf{N}-\mathbf{N}^{T} \mathbf{N S}+$ $\mathbf{N S N}^{T} \mathbf{I}$ - $\mathbf{S}$ equal to zero (for example, hydrostatic pressure), the effect of initial stress can be accounted through the asymmetry of the elastic tensor $B_{i j k l}$, as defined by relations (3). 

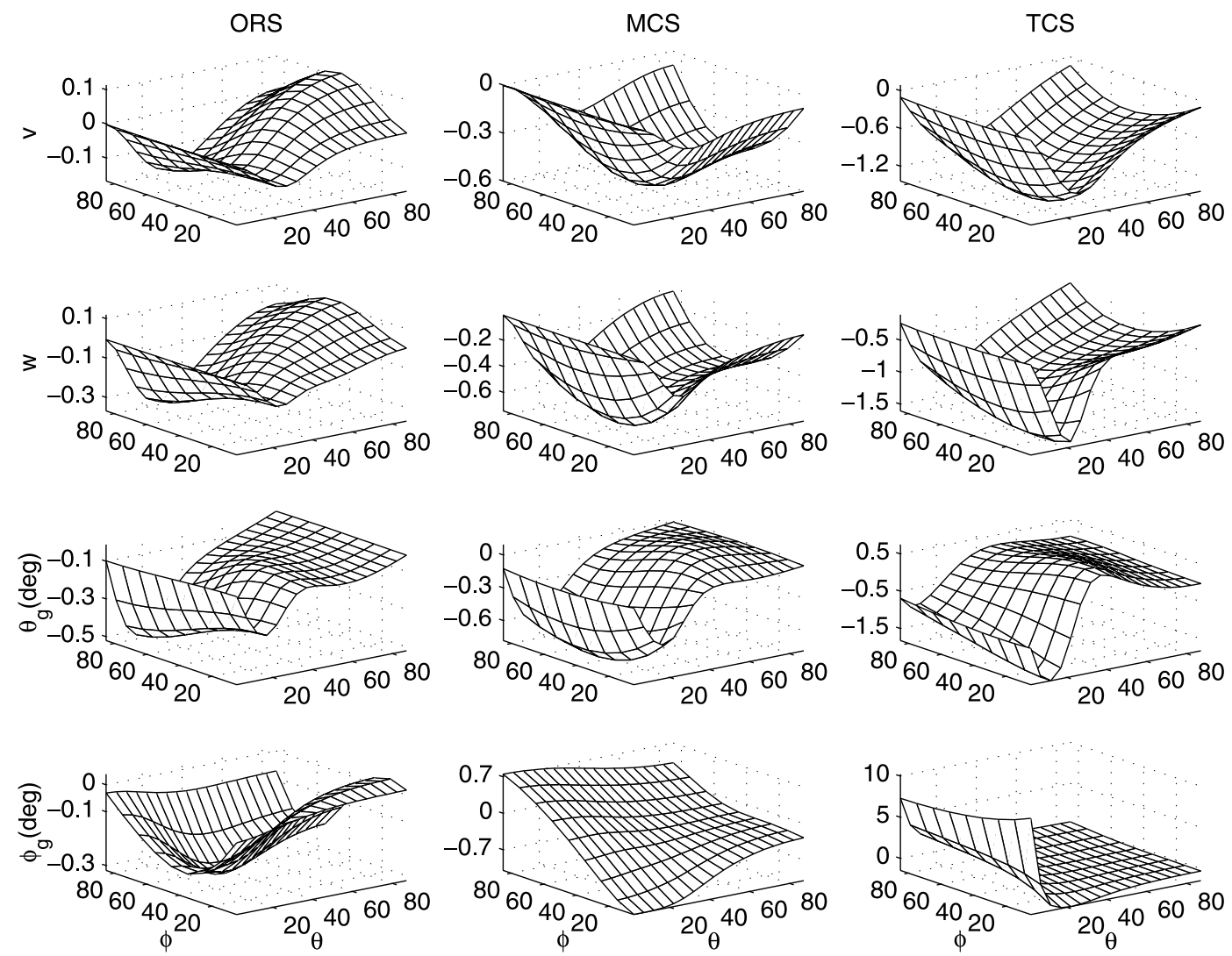

Figure 1. Variations of per cent deviations in phase velocity $(v)$ and group velocity $(w)$ and angular change in the ray direction $\left(\theta_{g}, \phi_{g}\right)$ of $q P$-wave for orthorhombic (ORS), monoclinic (MCS) and triclinic (TCS) anisotropies.

\section{Phase velocity}

Non-trivial solution of modified Christoffel equations (5) requires to satisfy an equation, given by

$$
\operatorname{det}(\mathbf{X}-h \mathbf{I})=0
$$

where $\mathbf{I}$ is the identity matrix of order three. This characteristic equation is, further, solved into a cubic equation in $h$, given by

$$
h^{3}+a h^{2}+b h+c=0
$$

where,

$$
\begin{aligned}
a= & -\left(X_{11}+X_{22}+X_{33}\right) \\
b= & X_{11} X_{22}+X_{11} X_{33}+X_{22} X_{33} \\
& -X_{12}^{2}-X_{13}^{2}-X_{23}^{2} \\
c= & X_{11} X_{23}^{2}+X_{22} X_{13}^{2}+X_{33} X_{12}^{2} \\
& -X_{11} X_{22} X_{33}-2 X_{12} X_{13} X_{23} .
\end{aligned}
$$

The three roots of this polynomial equation explain the propagation of three quasi-waves (Crampin 1981) in a pre-stressed anisotropic elastic medium. The symmetric matrix $\mathbf{X}$ in eigensystem (5) implies that all the three eigenvalues $(h)$ are real. The positive values of $h$ can be ensured through the values of initial stress components by making $\mathbf{X}$, a positive definite matrix. The symmetric matrix $\mathbf{X}$ will be positive definite when its, naturally ordered, principal minors will be positive (Hadley 1993). This provides non-linear relations among elastic constants, initial stress components and propagation direction. These relations may not be solved, analytically or numerically. Only, the verifications can be made for any given numerical model of a pre-stressed medium. Hence, the values of phase velocities of quasi-waves will be depending upon the values of initial stress components for every direction of propagation. Assuming that the initially-stressed medium is a non-attenuating one (i.e., all the anisotropic elastic constants are real numbers) the quasi-waves will be propagating with positive phase velocities. The real roots of equation (10), in descending order, are written as

$$
\begin{aligned}
h_{m}= & 2 \sqrt{H} \cos \left\{\frac{\psi+2 \pi(m-1)}{3}\right\}-a / 3, \\
& (m=1,2,3)
\end{aligned}
$$


ORS
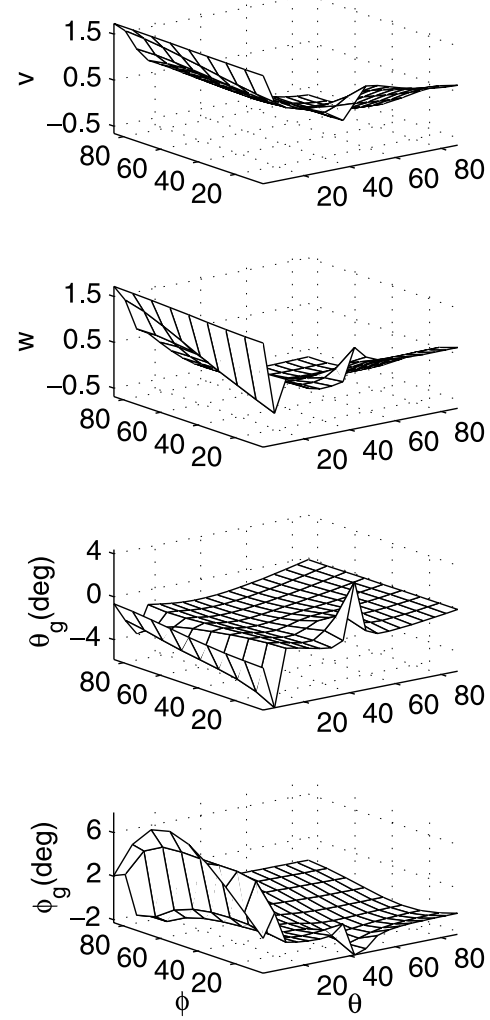

MCS
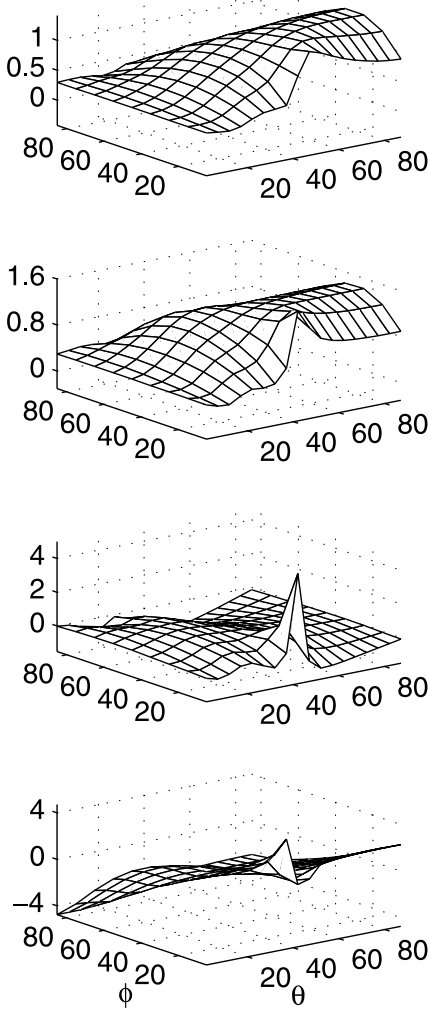

TCS
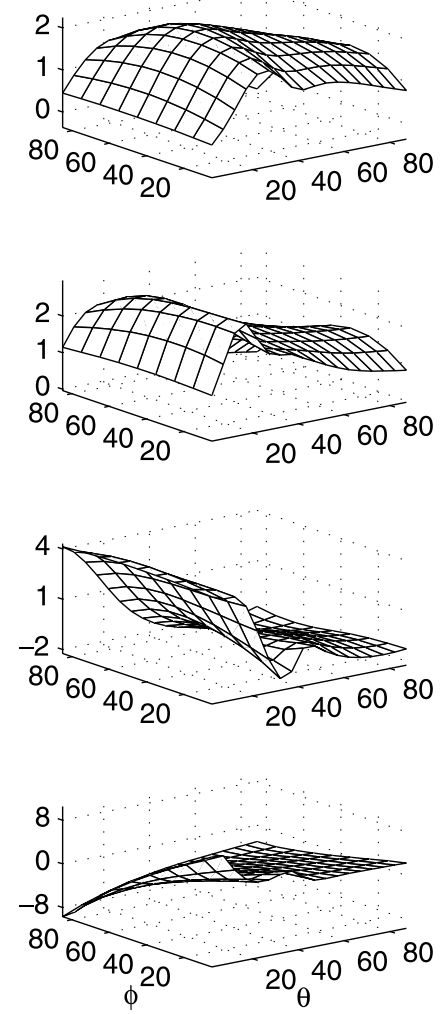

Figure 2. Variations of per cent deviations in phase velocity $(v)$ and group velocity $(w)$ and angular deviations in the ray direction $\left(\theta_{g}, \phi_{g}\right)$ of $q S 1$-wave for orthorhombic (ORS), monoclinic (MCS) and triclinic (TCS) anisotropies.

where,

$$
\begin{aligned}
\psi & =\tan ^{-1}\left(\frac{\Delta}{G}\right), \quad \Delta=\sqrt{\left(H^{3}-G^{2}\right)} ; \\
H & =\frac{a^{2}}{9}-\frac{b}{3}
\end{aligned}
$$

and

$$
G=\frac{\left(9 a b-27 c-2 a^{3}\right)}{54}
$$

$\left.v_{m}=\sqrt{(} h_{m}+\mathbf{N S N}^{T} / \rho\right),(m=1,2,3), \quad$ define the magnitudes of phase velocities of three non-attenuating quasi-waves propagating in the direction of (real) unit vector $N$. These waves, represented by $m=1,2$ and 3 , are called the $q P_{-}$, $q S 1$ - and $q S 2$-waves respectively; where $q S 1$ is the faster of the two split shear-waves (Crampin 1981).

\subsection{Special cases}

- In the absence of initial stress, the relations derived for wave propagation in pre-stressed anisotropic medium reduce to those derived in Sharma (2002). The reduced expressions explain the phase velocities of three quasi-waves in general anisotropic media. Further, for propagation in an isotropic elastic solid defined by two elastic constants $b_{11}$ and $b_{66}$, the relations (11) reduce to

$$
\begin{aligned}
& a=-\left(b_{11}+2 b_{66}\right), \quad b=b_{66}\left(b_{66}+2 b_{11}\right), \\
& c=-b_{11} b_{66}^{2},
\end{aligned}
$$

and, we have

$$
\begin{aligned}
H=\left[\frac{\left(b_{11}-b_{66}\right)}{3}\right]^{2}, & G & =\left[\frac{\left(b_{11}-b_{66}\right)}{3}\right]^{3}, \\
\Delta=0, & \psi & =0 .
\end{aligned}
$$

The three roots of cubic equation (10), in this case, are given by

$$
h_{1}=\frac{b_{11}}{\rho}, \quad h_{2}=h_{3}=\frac{b_{66}}{\rho} .
$$

This implies that the wave corresponding to $m=1$ in (12) reduce to $P$-wave and corresponding to $m=2,3$ reduce to $S$ wave.

- For propagation in an orthotropic medium in the presence of initial stress along principal 
ORS
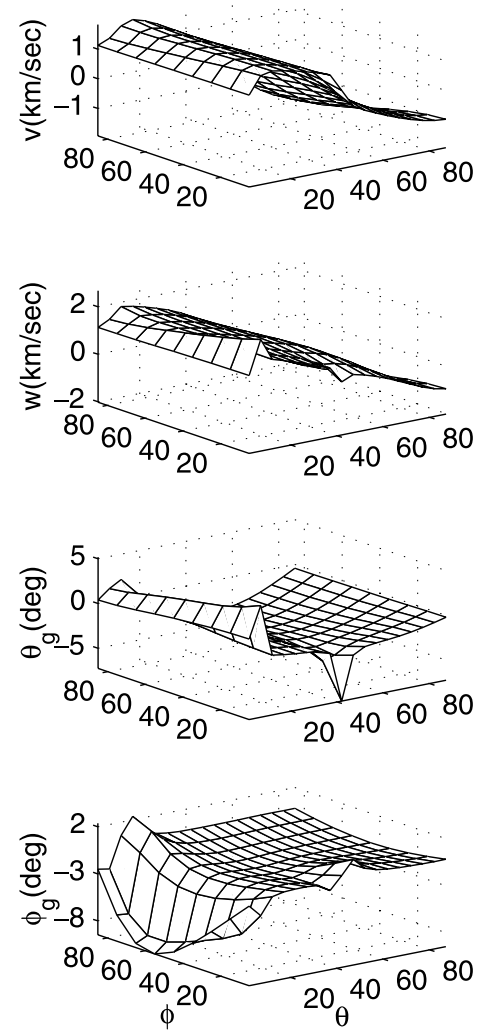

MCS
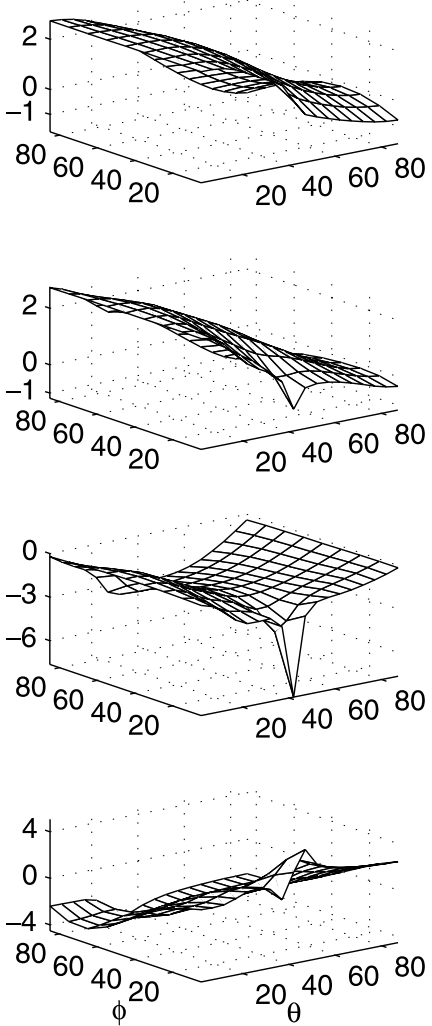

TCS
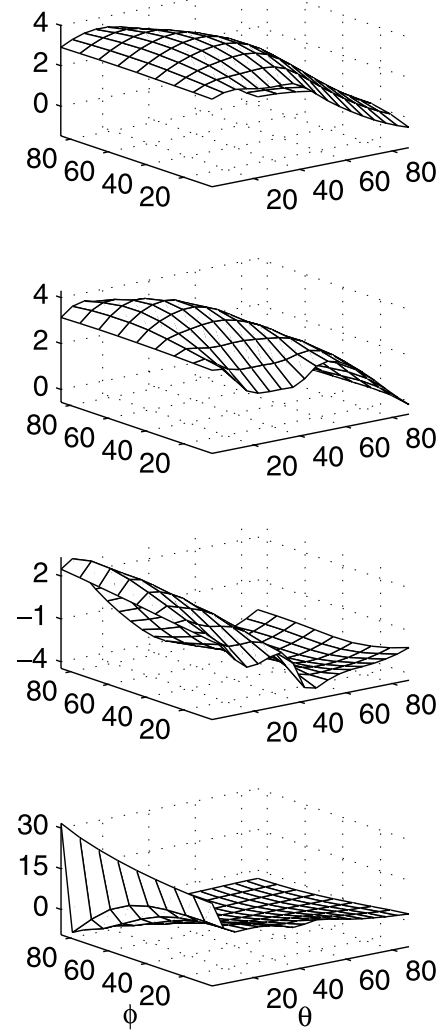

Figure 3. Variations of per cent deviations in phase velocity $(v)$ and group velocity $(w)$ and angular deviations in the ray direction $\left(\theta_{g}, \phi_{g}\right)$ of $q S 2$-wave for orthorhombic (ORS), monoclinic (MCS) and triclinic (TCS) anisotropies.

directions, the elements of symmetric matrix $X$, in (7), are given by

$$
\begin{aligned}
& X_{11}=b_{11} n_{1}^{2}+b_{66} n_{2}^{2}+b_{55} n_{3}^{2}-0.5 S_{11}, \\
& X_{22}=b_{66} n_{1}^{2}+b_{22} n_{2}^{2}+b_{44} n_{3}^{2}-0.5 S_{22} ; \\
& X_{33}=b_{55} n_{1}^{2}+b_{44} n_{2}^{2}+b_{33} n_{3}^{2}-0.5 S_{33}, \\
& X_{12}=\left[b_{12}+b_{66}+0.5\left(S_{11}-S_{22}\right)\right] n_{1} n_{2} ; \\
& X_{13}=\left[b_{13}+b_{55}+0.5\left(S_{11}-S_{33}\right)\right] n_{1} n_{3}, \\
& X_{23}=\left[b_{23}+b_{44}+0.5\left(S_{22}-S_{33}\right)\right] n_{2} n_{3} .
\end{aligned}
$$

Further, the phase velocities of three waves, as obtained from characteristic equation (9), are given by

$$
\begin{aligned}
v^{2}= & \frac{b_{11}}{\rho}, \quad \frac{\left[b_{66}+0.5\left(S_{11}-S_{22}\right)\right]}{\rho}, \\
& \frac{\left[b_{55}+0.5\left(S_{11}-S_{33}\right)\right]}{\rho},
\end{aligned}
$$

for propagation along $(1,0,0)$ direction and

$$
\begin{gathered}
v^{2}=\frac{b_{33}}{\rho}, \quad \frac{\left[b_{44}+0.5\left(S_{33}-S_{22}\right)\right]}{\rho}, \\
\frac{\left[b_{55}+0.5\left(S_{33}-S_{11}\right)\right]}{\rho},
\end{gathered}
$$

for propagation along $(0,0,1)$ direction. These velocity expressions are the same as those obtained in Tolstoy (1982). The corresponding results for isotropic medium with principal initial stresses are obtained through the substitutions of $b_{22}=b_{33}=b_{11}, b_{44}=b_{55}=b_{66}$, $b_{12}=b_{13}=b_{23}$ and $b_{12}=b_{11}-2 b_{66}$.

\section{Group velocity}

The energy associated with a quasi-wave travels with the group velocity along a ray at an angle to its phase direction. Mathematically, the group velocity and ray direction for a wave are derived from its phase direction and phase velocity. In anisotropic medium, phase and group velocity surfaces are, in general, different both in magnitude and direction. In a spherical co-ordinate 
system, let $v(\theta, \phi)$ define the phase velocity of a quasi-wave in a vertical plane along a direction making an angle $\theta$ with the polar (001) axis. Azimuth $\phi$ measures the orientation of vertical plane from 101-plane towards 011-plane. This defines $N=(\sin \theta \cos \phi, \sin \theta \sin \phi, \cos \theta)$. Following Ben-Menahem and Sena (1990), the group velocity of a quasi-wave ' $m$ ' is given by

$$
\mathbf{w}^{(m)}=v_{m} \widehat{e}_{r}+\frac{\partial v_{m}}{\partial \theta} \widehat{e}_{\theta}+\frac{1}{\sin \theta} \frac{\partial v_{m}}{\partial \phi} \widehat{e}_{\phi} .
$$

Ray direction or the direction of energy flux of a wave is determined from the components of its group velocity, which are expressed as follows:

$$
\begin{aligned}
& w_{x}=v \cos \phi \sin \theta+v_{, \theta} \cos \phi \cos \theta-v_{, \phi} \frac{\sin \phi}{\sin \theta} \\
& w_{y}=v \sin \phi \sin \theta+v_{, \theta} \sin \phi \cos \theta+v_{, \phi} \frac{\cos \phi}{\sin \theta} \\
& w_{z}=v \cos \theta-v_{, \theta} \sin \theta .
\end{aligned}
$$

The magnitude of the group velocity is

$$
w=\sqrt{v^{2}+\left(v_{, \theta}\right)^{2}+\left(\frac{v_{, \phi}}{\sin \theta}\right)^{2}},
$$

and ray direction $\left(\theta_{g}, \phi_{g}\right)$, in spherical co-ordinates, is given by

$$
\begin{aligned}
& \theta_{g}=\tan ^{-1}\left(\frac{\sqrt{w_{x}^{2}+w_{y}^{2}}}{w_{z}}\right) ; \\
& \phi_{g}=\tan ^{-1}\left(\frac{w_{y}}{w_{x}}\right) .
\end{aligned}
$$

For $h\left(=v^{2}-\mathbf{N S N}^{T} / \rho\right)$, the $v_{, \theta}$ and $v_{, \phi}$ in $(13)-(14)$ are defined by,

$$
v_{, j}=\frac{1}{2 v}\left(h_{, j}+2 \mathbf{N}_{, j} \mathbf{S N}^{T} / \rho\right) ; \quad(j=\theta, \phi) .
$$

The partial derivatives of $h$, derived, analytically from equation (10), are given by

$$
h_{, j}=-\frac{a_{, j} h^{2}+b_{, j} h+c_{, j}}{3 h^{2}+2 a h+b} .
$$

The partial derivatives of $\mathbf{N}$ are

$$
\begin{aligned}
& \mathbf{N}_{, \theta}=(\cos \theta \cos \phi, \cos \theta \sin \phi,-\sin \theta) ; \\
& \mathbf{N}_{, \phi}=(-\sin \theta \sin \phi, \sin \theta \cos \phi, 0) .
\end{aligned}
$$

\section{Numerical results and discussion}

The phase velocity, group velocity and ray direction are to be calculated for a given (arbitrary) phase direction. Medium chosen is Paragneiss, a general anisotropic crystalline rock. The density of the medium is $2727 \mathrm{~kg} / \mathrm{m}^{3}$. The elastic matrix (GPa) for Paragneiss (Rasolofosaon and Zinszner 2002) is given by

$$
\begin{array}{lll}
b_{11}=106.8 & b_{12}=27.10 & b_{13}=9.68 \\
b_{14}=-0.03 Z_{1} & b_{15}=0.28 Z_{1} & b_{16}=0.12 Z_{2} ; \\
b_{22}=99.00 & b_{23}=18.22 & b_{24}=1.49 Z_{1} \\
b_{25}=0.13 Z_{1} & b_{26}=-0.58 Z_{2} ; & b_{33}=54.57 \\
b_{34}=2.44 Z_{1} & b_{35}=-1.69 Z_{1} & b_{36}=-0.75 Z_{2} ; \\
b_{44}=25.97 & b_{45}=1.98 Z_{2} & b_{46}=0.43 Z_{1} \\
b_{55}=26.05 & b_{56}=1.44 Z_{1} & b_{66}=37.82 .
\end{array}
$$

$b_{i j}$ are the components of square matrix of order six and represent the elastic constant tensor $B_{i j k l}$ in two-suffix notation (Crampin 1989). The assumed values in matrix $\mathbf{S}=\left\{5,0.6 Z_{2}, 0.45 Z_{1}\right.$; $\left.0.6 Z_{2}, 5.5,0.5 Z_{1} ; 0.45 Z_{2}, 0.5 Z_{1}, 4.5\right\}$, defines the amount (in GPa) of initial stress in the medium. The values of $Z_{1}=Z_{2}=1$ define the anisotropic medium of arbitrary (triclinic) type. The values $Z_{1}=0, Z_{2}=1$ represent the monoclinic symmetry and $Z_{1}=Z_{2}=0$ represent the orthorhombic symmetry, in the anisotropic elastic medium considered.

Using the above numerical values, phase velocities of all the quasi-waves are calculated both in the presence and absence of initial stress. The difference between the two quantities represents the effect of presence of initial-stress on the phase velocities. Similarly, the effects of initial-stress are observed on group velocities and ray directions of quasi-waves. The phase direction $(\theta, \phi)$ is considered to be varying from $(0,0)$ to $\left(90^{\circ}, 90^{\circ}\right)$. Variations of these effects with phase direction are plotted in figures 1 to 3 . Deviations in velocities are calculated in per cent whereas the deviations in ray directions are computed in degrees. Details are as follows:

The first two rows of plots in figure 1 present the per cent change in phase and group velocities of $q P$-wave, due to the presence of initial stress. The other two rows show the directional variations of the changes (in degrees) in the ray direction of $q P$-wave due to the presence of initial stress. The first column of plots corresponds to the initial stress effect when the elastic medium is anisotropic with orthorhombic (ORS) symmetry. The second and third columns correspond to the media with monoclinic (MCS) and triclinic (TCS) symmetry 
of anisotropy, respectively. It is observed from the plots that phase velocities and group velocities are changing around one per cent. These changes are increasing with the anisotropy. The change in group velocity is slightly larger than in phase velocity. Change in the ray direction, however, increases with the anisotropy but it is not much significant. Only in triclinic anisotropy, the azimuth changes by about $7^{\circ}$. The directional variations of changes in the phase velocities, group velocities and raydirections of $q S 1$-wave and $q S 2$-wave are presented in figures 2 and 3, respectively. The general behaviours of the deviations in velocities and ray directions of these two waves are nearly similar to those for $q P$-wave. It is observed that the magnitude of the deviations for $q S 1$-wave is slightly more than for $q P$-wave. Similarly, the effect of the presence of initial stress on $q S 2$-wave is little more than on $q S 1$-wave. For example, in triclinic anisotropy the azimuth $\left(\phi_{g}\right)$ is changing up to $30^{\circ}$ whereas it is around $10^{\circ}$ for $q S 1$-wave.

The above discussion may be interpreted for the following results.

- The effect of initial stress on propagation increases with the increase of anisotropy in the elastic medium.

- Slower the wave, more is the effect of initial stress on its propagation.

- The effect of initial stress on the ray direction of every quasi-wave is observed mainly on its azimuth. This implies that studying the wave propagation in a fixed vertical plane may not be a right approach when medium is under initial stress.

\section{Conclusions}

The numerical discussion and results discussed above are obtained for a particular model with hypothetical initial stress values. So, these results may not qualify for generalisation. However, the mathematical model derived in this work may be used to compute the exact effects of initial stress on propagation. The anisotropy in the model should be viewed as means of investigating the internal constitution of the crust, and orientation/distribution of aligned seismic cracks. Initial stress in the model represents in situ stress distribution. When supported with a real/synthetic data, the mathematical model can be used for a variety of geophysical problems. Few of them may be explained as follows:

- The effect of confining stress on seismic velocities is used for pre-drill overpressure prediction (Sayers et al 2002) and for monitoring of hydrocarbon production in the boreholes.
- The seismic velocity measurements are used to estimate the crack parameters (Crampin et al 1980). Time lapse surveys in velocity measurements around a fault/reservoir may be used to predict the critical crack modifications there. These crack modifications in a focal region are believed to be the driving mechanism for the precursors of an earthquake. The crack modifications in/around a reservoir facilitate the flow of fluid in/across the reservoir. The derived velocity model provides a new approach to analyse crack dynamism with a coupled role of differential stress accumulated around a fault/ reservoir.

- Shear wave splitting due to extensive dilatancy anisotropy provides the basis for earthquake prediction and explains many other geological implications (Crampin 1987). By involving initial stress, it will give us new ways to examine in situ stress-geometry of the crust.

- It is the stress accumulation at a fault that can trigger an earthquake. Seismic velocity changes are the observable parameters of the stress accumulation in a part of the earth. The changes in ratio of $P$-wave velocity to $S$-wave velocity $\left(V_{P} / V_{S}\right)$ is an important precursor for earthquake prediction. The analysis of velocity anomalies through the pre-stressed mathematical model will enable to construct a more realistic mechanism of the earthquake preparation process. $\left(V_{P} / V_{S}\right)$ is also a key for direct seismic identification of pore-fluids.

- The characterization of residual stresses in the reservoirs, earth masses and composite materials could be possible through analysing the velocity variations in them. Otherwise, the stress is difficult to measure, in situ even in the most accessible parts of the crust.

- The anisotropic group velocity observations can be used to determine more accurate hypocentral locations of local earthquakes (Doyle et al 1982).

- The extent of fracturing in a region of a borehole is a vital factor in the extraction of oil and of geothermal heat. The observations of scattered waves provide the chief means of identification of the extent and nature of fractures. Involving initial stress, the changes monitored in reservoir evolution during hydrocarbon production and enhanced oil recovery may get more realistic interpretation.

- In the seismic frequency range, aligned fractures of rocks manifests itself in the form of anisotropy of $P$ - and $S$-wave velocities (Hudson 2001). The information on fracture distribution obtained from velocity inversion allows an estimation of the anisotropic permeability of the fracture rock system (Gibson and Toksoz 1990). 
The major cause of seismic anisotropy is the presence of cracks and fractures in the crustal rocks. Evolution and alignment of such cracks and fractures are commonly attributed by lithostatic stresses accumulated in the rocks. The fluidpressure in the saturated cracks is the main driving force in triggering an earthquake. Such a pressure also conducts the flow of fluid between the connected cracks in a reservoir. This signifies that initial stress and crack-induced anisotropy not only coexist in the crust but will, also, affect the quality/quantity of each other. The mathematical model derived in this work may be used to establish a theoretical relationship between effective stress and seismic signature of fracturing (Sinha et al 1995). The analytical expressions derived in this work can further, be used to study the effect of initial stress on the polarisation and scattering in a general anisotropic medium. The study may, in general, be useful in the non-destructive evaluation of materials and characterization of laminated composites.

\section{Acknowledgements}

Authors are thankful to the unknown reviewer for a descriptive review of the paper.

\section{Appendix A}

Consider a general anisotropic pre-stressed elastic medium represented by the elastic tensor $B_{i j k l}$ and density $\rho$. A non-symmetric square matrix of order six, $\mathbf{B}=\left\{b_{i j}\right\}$, is used to denote the elastic properties of the medium in two-suffix notations. The asymmetry of matrix $\mathbf{B}$ is explained through the relations:

$$
\begin{aligned}
& b_{12}-b_{21}=S_{22}-S_{11}, \quad b_{13}-b_{31}=S_{33}-S_{11}, \\
& b_{23}-b_{32}=S_{33}-S_{22} ; \\
& b_{14}-b_{41}=b_{24}-b_{42}=b_{34}-b_{43}=S_{23}, \\
& b_{15}-b_{51}=b_{25}-b_{52}=b_{35}-b_{53}=S_{13}, \\
& b_{16}-b_{61}=b_{26}-b_{62}=b_{36}-b_{63}=S_{12}, \\
& b_{54}=b_{45}, \quad b_{64}=b_{46}, \quad b_{65}=b_{56},
\end{aligned}
$$

where, $S_{i j},(i, j=1,2,3)$ are the components of initial-stress tensor.

Define a row matrix $\mathbf{N}=\left(n_{x}, n_{y}, n_{z}\right)$, where $n_{j}$ denotes the components of a unit vector normal to wave surface and, hence, it represents the direction of phase propagation. A square matrix $Z$ of order three is defined through its elements given by:

$$
\begin{aligned}
& Z_{11}=\mathbf{N A}_{\mathbf{1}} \mathbf{N}^{T}, \quad Z_{22}=\mathbf{N} \mathbf{A}_{\mathbf{2}} \mathbf{N}^{T}, \quad Z_{33}=\mathbf{N} \mathbf{A}_{\mathbf{3}} \mathbf{N}^{T}, \\
& Z_{12}=\mathbf{N} \mathbf{A}_{4} \mathbf{N}^{T}, \quad Z_{13}=\mathbf{N} \mathbf{A}_{\mathbf{5}} \mathbf{N}^{T}, \quad Z_{21}=\mathbf{N} \mathbf{A}_{\mathbf{6}} \mathbf{N}^{T}, \\
& Z_{23}=\mathbf{N A}_{\mathbf{7}} \mathbf{N}^{T}, \quad Z_{31}=\mathbf{N} \mathbf{A}_{\mathbf{8}} \mathbf{N}^{T}, \quad Z_{32}=\mathbf{N} \mathbf{A}_{\mathbf{9}} \mathbf{N}^{T},
\end{aligned}
$$

where $\mathbf{N}^{T}$ is the transpose of $\mathbf{N} . \mathbf{A}_{\mathbf{j}}, j=1,2, \ldots, 9$, the square matrices of order three, are defined as follows:

$$
\begin{aligned}
& \mathbf{A}_{\mathbf{1}}=\left\{b_{11}, b_{16}, b_{15} ; b_{61}, b_{66}, b_{65} ; b_{51}, b_{56}, b_{55}\right\} ; \\
& \mathbf{A}_{\mathbf{2}}=\left\{b_{66}, b_{62}, b_{64} ; b_{26}, b_{22}, b_{24} ; b_{46}, b_{42}, b_{44}\right\} ; \\
& \mathbf{A}_{\mathbf{3}}=\left\{b_{55}, b_{54}, b_{53} ; b_{45}, b_{44}, b_{43} ; b_{35}, b_{34}, b_{33}\right\} ; \\
& \mathbf{A}_{\mathbf{4}}=\left\{b_{16}, b_{12}, b_{14} ; b_{66}, b_{62}, b_{64} ; b_{56}, b_{52}, b_{54}\right\} ; \\
& \mathbf{A}_{\mathbf{5}}=\left\{b_{15}, b_{14}, b_{13} ; b_{65}, b_{64}, b_{63} ; b_{55}, b_{54}, b_{53}\right\} ; \\
& \mathbf{A}_{\mathbf{6}}=\left\{b_{61}, b_{66}, b_{65} ; b_{21}, b_{26}, b_{25} ; b_{41}, b_{46}, b_{45}\right\} ; \\
& \mathbf{A}_{\mathbf{7}}=\left\{b_{65}, b_{64}, b_{63} ; b_{25}, b_{24}, b_{23} ; b_{45}, b_{44}, b_{43}\right\} ; \\
& \mathbf{A}_{\mathbf{8}}=\left\{b_{51}, b_{56}, b_{55} ; b_{41}, b_{46}, b_{45} ; b_{31}, b_{36}, b_{35}\right\} ; \\
& \mathbf{A}_{\mathbf{9}}=\left\{b_{56}, b_{52}, b_{54} ; b_{46}, b_{42}, b_{44} ; b_{36}, b_{32}, b_{34}\right\}
\end{aligned}
$$

\section{References}

Ben-Menahem A and Sena A G 1990 Seismic source theory in stratified anisotropic media; J. Geophys. Res. 95 $15,395-15,427$.

Biot M A 1939 Non-linear theory of elasticity and the linearized case for a body under initial stress; Phil. Mag. $\mathbf{2 7}(\mathbf{7})$ 468-489.

Biot M A 1940 Influence of initial stress on elastic waves; J. Appl. Phys. 11 522-530.

Biot M A 1965 Mechanics of Incremental Deformation (New York: John Wiley) p. 214.

Cauchy A L 1827 Exercise de Mathematique, vol 2 (Paris: Bure Feres).

Chai J-F and Wu T-T 1996 Determination of surface wave velocities in a prestressed anisotropic solid; NDT $\&$ E Int. 29 281-292.

Crampin S 1981 A review of wave motion in anisotropic and cracked elastic media; Wave Motion 3 343-391.

Crampin S 1987 Geological and industrial implications of extensive dilatancy anisotropy; Nature 328 491-496.

Crampin S 1994 The fracture criticality of crustal rocks; Geophys. J. Int. 118 428-438.

Crampin S, McGonigle R and Bamford D 1980 Estimating crack parameters from observations of $P$ wave velocity anisotropy; Geophysics 45 345-360.

Degtyar A D and Rokhlin S I 1995 Absolute stress determination in orthotropic materials from angular dependence of ultrasonic velocities; J. Appl. Phys. 78 1547-1556. 
Degtyar A D and Rokhlin S I 1997 Stress effect on ultrasonic wave propagation through the solid-solid and liquid-solid plane interface; Reviews of Progress in Quantitative NDE Vol. 16B (eds) D O Thompson and D E Chimenti (New York: Plenum) pp. 1699-1706.

Degtyar A D and Rokhlin S I 1998 Stress effect on boundary conditions and elastic wave propagation through an interface between anisotropic media; J. Acoust. Soc. Am. 104 1992-2003.

Doyle M, McGonigle R and Crampin S 1982 The effects of crack anisotropy on the hypocentral locations of local earthquakes; Geophys. J. R. Astr. Soc. 69 137-157.

Gibson Jr. R L and Toksoz M N 1990 Permeability estimation from velocity anisotropy in fractured rocks; J. Geophys. Res. 95 15,643-15,656.

Hadley G 1993 Linear Algebra (New Delhi: Narosa) pp. 260-262.

Hayes M 1963 Wave propagation and uniqueness in prestressed elastic solids; Proc. Roy. Soc. London A274 500-506.

Hudson J A 2001 Effect of fluid pressure on wave speeds in cracked solids; Geophys. J. Int. 143 302-310.

Man C-S and Lu W Y 1987 Towards an acoustoelastic theory for measurements of residual stress; J. Elast. 17 159-182.
Norris A N and Sinha B K 1995 Speed of a wave along a fluid/solid interface in the presence of anisotropy and prestress; J. Acoust. Soc. Am. 98 1147-1154.

Prikazchikov D A and Rogerson G A 2003 Some comments on the dynamic properties of anisotropic and strongly anisotropic pre-stressed elastic solids; Int. J. Engg. Sci. 41 149-171.

Rasolofosaon P N J and Zinszner B E 2002 Comparison between permeability anisotropy and elasticity anisotropy of reservoir rocks; Geophysics 67 230-240.

Sayers C M, Johnson G M and Denyer G 2002 Predrill pore-pressure prediction using seismic data; Geophysics 67 1286-1292.

Sharma M D and Gogna M L 1991 Propagation of Love waves in an initially stressed medium consisting of slow elastic layer lying over a liquid-saturated porous solid half-space; J. Acoust. Soc. Am. 89 2584-2588.

Sharma M D 2002 Group velocity in general direction in a general anisotropic medium; Int. J. Solids \& Struct. 39 $3277-3288$.

Sinha B K, Kostek S and Norris A N 1995 Stoneley and flexural modes in pressurised boreholes; J. Geophys. Res. $10022,375-22,381$.

Tolstoy I 1982 On elastic waves in prestressed solid; J. Geophys. Res. 87 6823-6827. 